\title{
Asymptotic Strategy for Matching Homogenized Structures. Conductivity Problem
}

\author{
Alexander G. Kolpakov ${ }^{1}$, Igor V. Andrianov ${ }^{2}$, and Danila A. Prikazchikov ${ }^{3}$
}

\author{
${ }^{1}$ SysAn, A. Nevskogo, 12-A, 34, Novosibirsk 630075, Russia \\ e-mail: algk@ngs.ru \\ ${ }^{2}$ Institute of General Mechanics, RWTH Aachen University, Templergraben 64, D-52056, Aachen, \\ Germany \\ e-mail: igor.andrianov@gmail.com \\ ${ }^{3}$ School of Computing and Mathematics, Keele University, Keele, Staffordshire, ST5 5BG, UK \\ e-mail: d.prikazchikov@keele.ac.uk
}

\begin{abstract}
The paper is concerned with application of the homogenization theory to bodies containing macroinhomogeneities or bodies, parts of which cannot be homogenized (partial homogenization). This situation arises, in particular, for problems of joining homogeneous and periodically inhomogeneous bodies, or combining inhomogeneous bodies of different periodic structure. The peculiarity of the problem is related to a boundary layer, possibly arising on the interface of the matched components. Moreover, this boundary layer may be either real or fictitious, with the latter occurring due to inaccurate formulation of boundary conditions along the interface, ignoring the effect of the micro-stresses. The consideration is carried out within the framework of the steadystate heat equation. The focus of current investigation is on formulation of the problem for the periodicity cell in case of discontinuous homogenized deformations, when these cannot be treated as independent of the "fast" variables. The first order correctors are constructed. The issue of consistent matching procedure, avoiding emergence of fictitious boundary layers, is discussed. It is shown that the temperature of an inhomogeneous fragment on the boundary may be determined from the solution of the homogenized problem, whereas the derivatives (temperature gradients) require fast correctors of the homogenization theory to be taken into account. The analytical consideration is confirmed by results of numerical simulations.
\end{abstract}

Keywords: thermal conductivity problem, steady-state heat equation, contact of periodic structures, homogenization theory, fast corrector, boundary layer 


\section{Introduction}

Homogenization theory is an excellent tool, when the considered system is periodic or quasiperiodic. Within the framework of the conventional homogenization theory the formulations for the periodicity cells and the homogenized problem usually rely on the smoothness of the limiting problem solution and its derivatives. In this paper we are dealing with more difficult cases, when it is impossible to perform "separation of variables" (e.g. [1]), crucial for classical homogenization, because the derivatives of the homogenized solution are not independent of the fast variables. Indeed, the periodicity can be violated, a situation typically occurring in the vicinity of the boundary of finite domains, due to various defects as cracks or holes or inclusions, or near the interface of two bodies of different structure, see Fig 1. The first case corresponds to a body containing inhomogeneities on the macro-level, whereas the second case is related to a problem of joining the micro- and macrostructure.

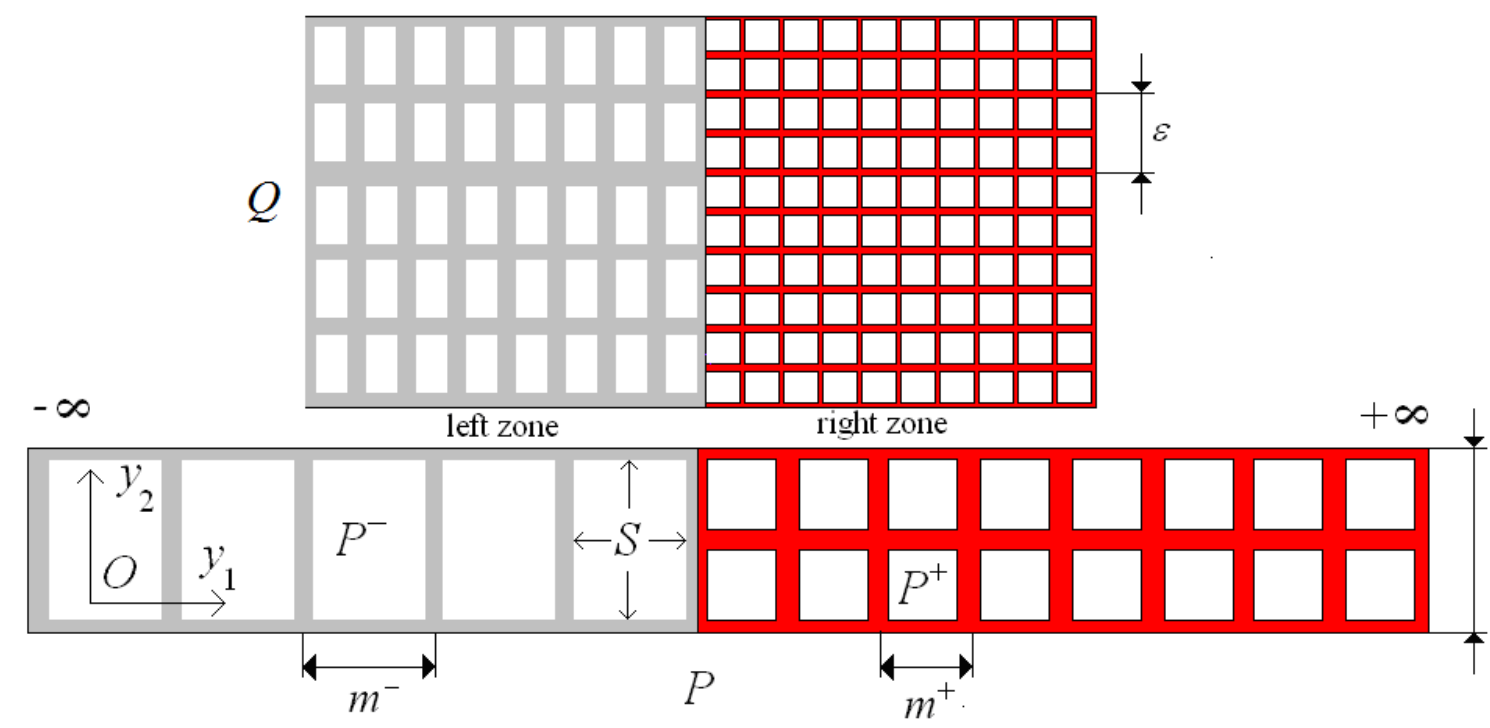

Figure 1. The body $Q$, containing two parts with different microstructure (top) and their periodicity cell $P$ (bottom), consisting of two parts $P^{-}$and $P^{+}$.

Therefore, we arrive at a non-trivial problem of matching homogenized solutions or dealing with homogenized solution which contains macro-inhomogeneities.

Existing homogenization theories allow correct joining of homogenized solutions on the macro-level. For example, the statement of the associated problem of heat transfer between the two structures shown in Fig. 1 should include continuity conditions for homogenized temperature together with the averaged heat flux in the normal direction. At the same time, attempts of 
matching the same structures on the micro-level, i.e. not applying homogenization, lead to a rather non-trivial problem, requiring separate consideration.

There have been a large number of contributions on the subject, see e.g. [2, 3] and references therein for a small representative sample. From a physical point of view, the problem is related to coupling between either, non-local and local continuum mechanics [4], or discrete and continuum models (atomistic-to-continuum coupling) [5-9], or, possibly, local and global models [10]. As a rule, the proposed approach relies on the so-called bridging (overlap) domains ("gluing zones", "handshake region"), see e. g. [11]. As noted in [2], these methods are closely related to the overlapping Schwarz methods [12] stemming from the classical alternating Schwarz algorithm. The main idea of these methods can be described as follows: part of the system in the vicinity of the defect or boundary is considered within the framework of discrete media, whereas the rest is assumed to be homogenized.

Within the overlap domain, the discrete and homogenized solutions should match in some sense. For example, the discrete solutions may be interpolated (or continuous solutions could be discretized). "In this transition region, approximations are made such as treating finite element nodes as atoms, or vice versa, to accommodate the incompatibility between a non-local atomistic description and a local finite element description" [9]. Linear interpolation was used in [5] for addressing this issue, however, other types of interpolation could also be applied. Gluing of local and global solutions may also rely on energy method [2]; another option is the Lagrange multiplier method or augmented Lagrangian method [8].

A problem for a 2D fibre-reinforced composite containing a defected fibre has been studied in [13]. This fibre was considered within the discrete framework, with the rest of the fibres being homogenized. Then, matching was performed through the concept of a bridging domain, leading to an approximate analytical solution of a rather technical problem.

An alternative approach involves formulation of certain artificial boundary conditions on the interface between discrete and continuous domains. As mentioned in [14], numerical simulations of crystal defects are necessarily restricted to finite computational domains, supplying artificial boundary conditions that emulate the effect of embedding the defect in an effectively infinite crystalline environment. Hence, the question of accuracy of boundary conditions arises. The issue of artificial boundary conditions is thoroughly addressed in [15], studying vibrations of one- and two-mass chains with defects. According to the procedure, the part which is outside the defect zone is replaced by its continuous analogue, with the defect modeled through certain boundary conditions. Since the discrete problem and its continuous model both possess exact solutions, the efficiency of artificial boundary conditions may be readily verified. The approach is extended further in [16], investigating vibrations of 1+1D composites with defects. 
The problem of a contact between two periodic micro-inhomogeneous media was first considered in [18] within the framework of homogenization theory. The asymptotic approximation of solution was obtained using the boundary layer theory in $[19,20]$. The existence and uniqueness was investigated, along with the structure of asymptotic approximations and limiting problems. A notable contribution to analysis of boundary layers in the vicinity of interfaces between slender and continuous bodies was made in [21]. The theoretical results obtained in [21], correlate with our numerical analysis for a structure combining a homogeneous body and a coarse lattice, see also [22].

In practice the described method of bridging domain and that of artificial boundary conditions deliver reasonable results. However, it should be noted, that there are still open questions regarding rigorous approximation of solution in the method of bridging (overlap) domains, or justified choice of appropriate artificial boundary conditions. Therefore, development of a mathematical theory, allowing accurate and natural matching of solutions in homogenized domains with solutions in local or homogeneous domains, is of clear interest. One of the ways to achieve this would be incorporation of an additional asymptotic expansion term (boundary layer correctors), similarly to boundary layers arising within the framework of classical homogenization [17]. The current paper aims at the construction of such a theory, with the consideration below carried out for a steady-state problem of heat transfer.

The paper is organized as follows. In Sect. 2 we deal with the problem of homogenization, with the specific focus on the transition region between a homogeneous and periodically inhomogeneous media. In Sect. 3 the problem of correct joining between the discrete (or initially continuous) and homogenized parts of the body is investigated. Finally, concluding remarks are presented in Sect. 4.

\section{Homogenization and local field on the interface of homogeneous and periodically inhomogeneous media}

Consider a 2D problem of steady-state heat transfer in an inhomogeneous periodic medium, occupying the domain $Q$, with $\varepsilon<<1$ being a typical size of the periodicity cell. The original formulation of the problem is given by

$$
\int_{Q} a(\mathbf{x} / \varepsilon) \nabla u(\mathbf{x}) \nabla \varphi(\mathbf{x}) d \mathbf{x}=\int_{Q} q(\mathbf{x}) \varphi(\mathbf{x}) d \mathbf{x}
$$

where $a(\mathbf{x} / \varepsilon)$ are local coefficients of thermal conductivity, $u(\mathbf{x})$ is the sought for solution, $\varphi(\mathbf{x})$ denotes a trial function, and $q(\mathbf{x})$ is the heat flow. 
It should be noted that the local characteristics $a(\mathbf{x} / \varepsilon)$ of composite materials are usually piecewise continuous. In our consideration, even if $a(\mathbf{x} / \varepsilon)$ are continuous, the homogenized characteristics may be piecewise continuous, which corresponds to the "interface" or "diffraction" problem, see [23], and also [24]. In fact, the interface problem (1) can be equivalently represented by the appropriate equations with constant coefficients (namely, for every component of structure), along with conditions of perfect bonding at the interfaces. After [23], it is widely accepted that the adequate technique for analysis of such problems is based on the variational (weak) formulation. Taking into account that the homogenization method is also based on the weak formulation of the problem, we prefer to use the form (1).

Equation (1) must be supplemented with the boundary conditions at the external boundary $\partial Q$ of the domain $Q$. At the same time, in this study we are not concerned with the effects of the heat sources and boundary conditions, focusing entirely on the behavior of solution in the nearinterface vicinity between the media. Moreover, as will be shown later, even though the boundary layer may potentially exist in the vicinity of the joint, it is a phenomenon of different nature, not related to homogenization.

Following the conventional homogenization technique, the solution is now sought for in the form

$$
u=u_{0}(\mathbf{x})+\varepsilon u_{1}(\mathbf{x}, \mathbf{x} / \varepsilon) .
$$

Function $u_{0}(\mathbf{x})$ describes the "slow" (the macroscopic) component of the displacements and $\varepsilon u_{1}(\mathbf{x} / \varepsilon)$ is corrector. We remark that the corrector is of order $\varepsilon$, but the gradient of corrector $\nabla_{x} \varepsilon u_{1}(\mathbf{y})=\nabla_{x} \varepsilon u_{1}(\mathbf{x} / \varepsilon)=\nabla_{y} u_{1}(\mathbf{x} / \varepsilon)$ is of order unity $\left(\nabla_{x}\right.$ and $\nabla_{y}$ denote gradients in $\mathbf{x}$ and $\mathbf{y}$, respectively). Hereafter, we use notation $\mathbf{y}=\left(y_{1}, y_{2}\right)=\mathbf{x} / \varepsilon$ for the "fast" variable.

The form of the corrector $\varepsilon u_{1}(\mathbf{y})$ is chosen depending on the geometry of the joint bodies (see Fig. 1). Naturally, it is assumed as a sum of periodic components, along with the boundary layer terms. Due to periodicity of the whole body with respect to coordinate $y_{2}$, the periodic components of the solution have the same period in $y_{2}$, say 1 . As for $y_{1}$-coordinate, the periodic parts of the solution have the periods $m^{-}$and $m^{+}$, on the left and right from the joint, respectively. The boundary layer-type components decay rapidly away from the joint, in our case, as $y_{1} \rightarrow \pm \infty$. Thus, as $y_{1} \rightarrow \pm \infty$, the corrector $\varepsilon u_{1}(\mathbf{y})$ approaches the functions, periodic in $y_{1}$ with periods $m^{-}$ and $m^{+}$.

The trial function has a similar representation to (2), i.e. 


$$
\varphi=\varphi_{0}(\mathbf{x})+\varepsilon \varphi_{1}(\mathbf{x}, \mathbf{y})
$$

On inserting (2) and (3) into (1), one arrives at a problem for $u_{1}(\mathbf{y})$

$$
\int_{P} a(\mathbf{y})\left(\nabla_{y} u_{1}+\nabla_{x} u_{0}\right) \nabla_{y} \varphi_{1} d \mathbf{x}=0
$$

for more detail see [17]. The formulation (4) is obtained through separation of "slow" and "fast" variables, $\mathbf{x}$ and $\mathbf{y}$, respectively, along with the differential relation [1]

$$
\nabla_{x} f(\mathbf{x}, \mathbf{y})=\nabla_{x} f(\mathbf{x}, \mathbf{y})+\varepsilon^{-1} \nabla_{y} f(\mathbf{x}, \mathbf{y}) .
$$

Equation (4) leads to the following problem for the periodicity cell

$$
\begin{aligned}
& \left(a_{i j}(\mathbf{y}) u_{1, j y}+a_{i 1}(\mathbf{y}) \frac{\partial u_{0}(\mathbf{x})}{\partial x_{1}}+a_{i 2}(\mathbf{y}) \frac{\partial u_{0}(\mathbf{x})}{\partial x_{2}}\right)_{, i y}=0 \text { in } P, \\
& \left(a_{i j}(\mathbf{y}) u_{1, j y}+a_{i 1}(\mathbf{y}) \frac{\partial u_{0}(\mathbf{x})}{\partial x_{1}}+a_{i 2}(\mathbf{y}) \frac{\partial u_{0}(\mathbf{x})}{\partial x_{2}}\right) n_{j}=0 \text { at } S,
\end{aligned}
$$

where the quantity $u_{1}(\mathbf{y})$ is a periodic function along $y_{2}$ (with period 1 ), and tends to a periodic function with periods $m^{ \pm}$along $y_{1}$ in the limits $y_{1} \rightarrow \pm \infty$. Hereinafter we denote $u_{, j y}=\frac{\partial u}{\partial y_{j}}$, $u_{, j x}=\frac{\partial u}{\partial x_{j}} ; S$ is a free surface of the periodicity cell $P$, see Fig. 1 (allowing to account for pores, holes, etc. ), and $\mathbf{n}$ is the outer normal to $S$.

Due to linearity, the problem (5) may be separated into two sub-problems involving the terms $a_{i 1}(\mathbf{y}) \frac{\partial u_{0}(\mathbf{x})}{\partial x_{1}}$ and $a_{i 2}(\mathbf{y}) \frac{\partial u_{0}(\mathbf{x})}{\partial x_{2}}$ instead of their sum. It is crucial for the conventional homogenization procedure that the quantities of the problem (5) depend on the fast variables $\mathbf{y}$, whereas the derivatives $\frac{\partial u_{0}(\mathbf{x})}{\partial x_{1}}$ and $\frac{\partial u_{0}(\mathbf{x})}{\partial x_{2}}$ depend on the slow variables $\mathbf{x}$. Therefore, in analysis of (5) the quantities $\frac{\partial u_{0}(\mathbf{x})}{\partial x_{1}}$ and $\frac{\partial u_{0}(\mathbf{x})}{\partial x_{2}}$ may be treated as constants. Hence, the separation into slow and fast variables is performed, leading to

$$
u_{1}(\mathbf{y})=N^{i}(\mathbf{y}) \frac{\partial u_{0}(\mathbf{x})}{\partial x_{i}}
$$


where $N^{i}(\mathbf{y})$ are certain functions of the fast variables, see [1] (here and below, summation over repeated indices is assumed).

Now we can observe the difference from the conventional homogenization procedure for macro-homogeneous media, when the homogenized material characteristics are continuous and the homogenized solutions are smooth. In order for problem (5) to have solution in the form (6), the gradient $\nabla_{x} u_{0}(\mathbf{x})$ should be independent of the fast variables, so it should be continuous along the slow variables. In our case the quantity $\nabla_{x} u_{0}(\mathbf{x})$ is discontinuous.

Then, the gradient $\nabla_{x} u_{0}(\mathbf{x})$ is represented as

$$
\nabla_{x} u_{0}(\mathbf{x})=\nabla_{x} u_{0}^{-}(\mathbf{x}) \chi^{-}(\mathbf{x})+\nabla_{x} u_{0}^{+}(\mathbf{x}) \chi^{+}(\mathbf{x})
$$

where $\chi^{-}(\mathbf{x})$ and $\chi^{+}(\mathbf{x})$ are indicator functions of the domains - and + , respectively. Due to presence of step indicator functions in (7), this gradient cannot be constant over the joint region.

Let us analyze the problem (5) for the periodicity cell. It should be emphasized that not all of the derivatives of the homogenized solution $u_{0}(\mathbf{x})$ are discontinuous. Indeed, since the solution of the homogenized problem $u_{0}(\mathbf{x})$ is continuous, its values from the left and right of the joint, $u_{0}^{-}(\mathbf{x})$ and $u_{0}^{+}(\mathbf{x})$, respectively, coincide. In other words, the jump over the boundary $\left[u_{0}(\mathbf{x})\right]=u_{0}^{+}(\mathbf{x})-u_{0}^{-}(\mathbf{x})$ is zero

$$
\left[u_{0}(\mathbf{x})\right]=0 .
$$

On differentiating (8) with respect to $x_{2}$, i.e. along the joint, we deduce that

$$
\frac{\partial}{\partial x_{2}}\left[u_{0}(\mathbf{x})\right]=\left[\frac{\partial u_{0}(\mathbf{x})}{\partial x_{2}}\right]=0
$$

implying that the derivative with respect to $x_{2}$ is continuous. Hence, the representation (7) is not required for the derivative $\frac{\partial u_{0}(\mathbf{x})}{\partial x_{2}}$, so it may be treated as a constant, exactly as in case of the classical homogenization.

Now, let us consider the problem for the periodicity cell associated with the derivative $\frac{\partial u_{0}}{\partial x_{1}}$ and discuss the decomposition of the solution into periodic and boundary layer components. First, we note that $\frac{\partial u_{0}}{\partial x_{1}}$ is discontinuous. The associated problem for the periodicity cell is given by 


$$
\begin{aligned}
& \left(a_{i j}(\mathbf{y}) M_{, j y}+a_{i 1}(\mathbf{y}) u_{0,1 x}^{-}(\mathbf{x}) \chi^{-}(\mathbf{x})+a_{i 1}(\mathbf{y}) u_{0,1 x}^{+}(\mathbf{x}) \chi^{+}(\mathbf{x})\right)_{, i y}=0, \quad \text { in } P, \\
& \left(a_{i j}(\mathbf{y}) M_{, j y}+a_{i 1}(\mathbf{y}) u_{0,1 x}^{-}(\mathbf{x}) \chi^{-}(\mathbf{x})+a_{i 1}(\mathbf{y}) u_{0,1 x}^{+}(\mathbf{x}) \chi^{+}(\mathbf{x})\right) n_{j}=0, \quad \text { at } S,
\end{aligned}
$$

where $M(\mathbf{y})$ is periodic with period 1 along $y_{2}$ and tends to a periodic functions with periods $m^{ \pm}$ along $y_{1}$ in the limits $y_{1} \rightarrow \pm \infty$. Clearly, problem (10) follows from (5), when only one of the derivatives of the homogenized solution, namely, $\frac{\partial u_{0}}{\partial x_{1}}$ is not zero, and this derivative is represented as (7).

In the presence of discontinuous terms $a_{i 1}(\mathbf{y}) u_{0,1 x}^{-}(\mathbf{x}) \chi^{-}(\mathbf{x})+a_{i 1}(\mathbf{y}) u_{0,1 x}^{+}(\mathbf{x}) \chi^{+}(\mathbf{x})$, it is convenient to treat the boundary value problem (10) in the weak sense.

Clearly, solutions of the problems

$$
\begin{gathered}
\left(a_{i j}(\mathbf{y}) M_{, j y}+a_{i 1}(\mathbf{y}, \mathbf{x})\right)_{, i y}=0 \quad \text { in } P^{ \pm}, \\
\left(a_{i j}(\mathbf{y}) M_{, j y}+a_{i 1}(\mathbf{y}, \mathbf{x})\right) n_{j}=0 \text { at } S,
\end{gathered}
$$

in the right $\left(P^{+}: y_{1}>0\right)$ and left $\left(P^{-}: y_{1}<0\right)$ domains are solutions of the periodicity cell problems within the framework of conventional homogenization theory for periodic media located to the left and to the right from the interface, see Fig. 1.

Let us introduce the auxiliary function

$$
v(\mathbf{x}, \mathbf{y})=u_{0,1 x}^{-}(\mathbf{x}) N^{1-}(\mathbf{y}) \chi^{-}(\mathbf{x})+u_{0,1 x}^{+} N^{1+}(\mathbf{y}) \chi^{+}(\mathbf{x}) .
$$

Then, the function $M(\mathbf{y}, \mathbf{x})$ can be decomposed in terms of the periodic component $v(\mathbf{y}, \mathbf{x})$ and boundary layer $m(\mathbf{y})$

$$
M(\mathbf{y}, \mathbf{x})=m(\mathbf{y})+v(\mathbf{y}, \mathbf{x}) .
$$

Hence, (11) is transformed to the following problem for $m(\mathbf{y})$

$$
\begin{gathered}
\left(a_{i j}(\mathbf{y}) m_{, j y}\right)_{, i y}=0 \quad \text { in } P, \\
a_{i j}(\mathbf{y}) m_{, j y} n_{j}=0 \quad \text { at } S,
\end{gathered}
$$

where $m(\mathbf{y})$ is periodic with period 1 along $y_{2}$ and $m(\mathbf{y}) \rightarrow 0$ in the limits $y_{1} \rightarrow \pm \infty$.

It should be noted that the function (12) is discontinuous. Therefore, the following jump conditions should be imposed on the joint $P \cap\left\{y_{1}=0\right\}$

$$
[m]=\left[u_{0,1 x}^{-}(0) N^{1+}(\mathbf{y})+u_{0,1 x}^{+}(0) N^{1-}(\mathbf{y})\right],
$$




$$
\left[a_{i j} m_{, j y}\right] n_{j}=\left(a_{i j}(\mathbf{y}) N_{, j y}^{1+}(\mathbf{y}) u_{0,1 x}^{+}(0)-a_{i j}(\mathbf{y}) N_{, j y}^{1-}(\mathbf{y}) u_{0,1 x}^{-}(0)\right) n_{j},
$$

with $u_{0,1 x}^{-}(0)$ and $u_{0,1 x}^{+}(0)$ denoting the values of the functions $u_{0,1 x}^{-}(\mathbf{x})$ and $u_{0,1 x}^{+}(\mathbf{x})$ on the interface $x_{1}=0$.

The condition (16) has a physical interpretation of a jump of the heat flux

$$
\left[\boldsymbol{\sigma}_{n}\right]=\boldsymbol{\sigma}_{n}^{+}(\mathbf{y}) u_{0,1 x}^{+}(0)-\boldsymbol{\sigma}_{n}^{-}(\mathbf{y}) u_{0,1 x}^{-}(0) \text {. }
$$

The average value of the right hand side of (17) along the joint $P \cap\left\{y_{1}=0\right\}$ is equal to zero, which leads to fast decay of solution at a distance from the joint (it is scalar analog of the Saint-Venant principle for self-equilibrated loads, see e. g. [23, 24]). As noted above, the function $m(\mathbf{y})$ is "gluing" the solution (12) from the left and right sides of the joint, i.e. acts as a boundary layer localized over the transition region.

In order to illustrate the proposed methodology above let us consider a numerical example of joining a homogeneous body and a lattice, see Fig. 2.

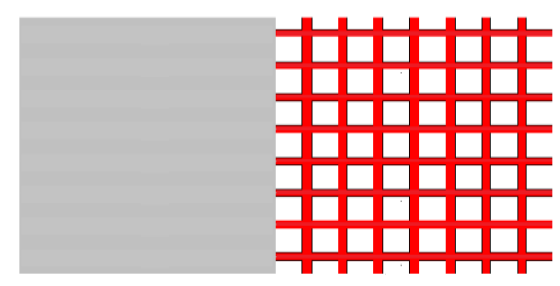

Figure 2. A system containing a homogeneous body and a lattice.

It is convenient to separate the periodicity cells as shown in Fig. 3. Below we assume that the contact occurs precisely on the boundaries of the periodicity cells (this restriction is not crucial for the method and is only taken in order to simplify the calculations).

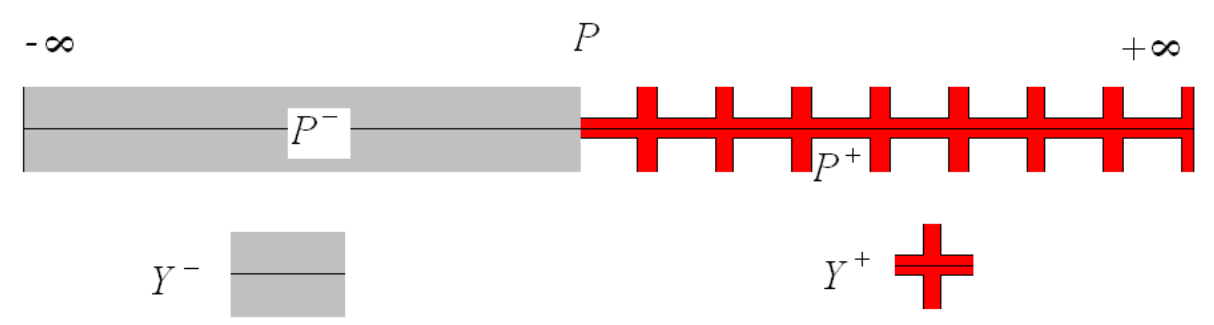

Figure 3. The periodicity cell for a joint body, including the periodicity cells of the left and right components. 


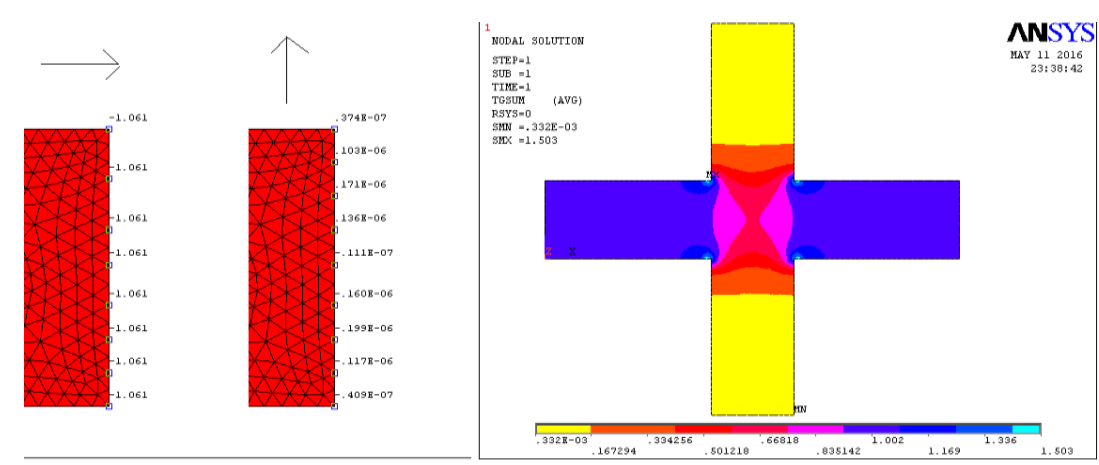

Figure 4. Solution of the problem for the periodicity cell for the lattice.

The solution of the problem for the right component $Y^{+}$of the elementary cell has been simulated in ANSYS, see Fig. 4. It may be observed that the normal flux and temperature are virtually constant along the boundary of the cell $Y^{+}$, having $\boldsymbol{\sigma}_{n}^{+}=(1.061,0)$. In the present case, due to symmetry of the elementary cell (with respect to the $90^{\circ}$ rotation around the origin), the homogenized material is isotropic, with the homogenized coefficients of thermal conductivity equal to 0.20012.

On the boundary of the elementary cell $Y^{-}$we have $\boldsymbol{\sigma}_{n}^{-}=(1,0)$ and constant temperature. Therefore,

$$
\begin{aligned}
& {[m(\mathbf{y})]=\left[u_{0,1 x}^{-}(\mathbf{x}) N^{1+}(\mathbf{y})+u_{0,1 x}^{+} N^{1-}(\mathbf{y})\right]=0,} \\
& {\left[a_{i j}(\mathbf{y}) m_{, j y}\right] n_{j}=\left(a_{i j}(\mathbf{y}) N_{, j y}^{1+}(\mathbf{y}) u_{0,1 x}^{+}(\mathbf{x})-a_{i j}(\mathbf{y}) N_{, j y}^{1-}(\mathbf{y}) u_{0,1 x}^{-}(\mathbf{x})\right) n_{j} .}
\end{aligned}
$$

The problem for the boundary layer component $m(\mathbf{y})$ takes the form

$$
\begin{aligned}
& \left(a_{i j}(\mathbf{y}) m_{, j y}\right)_{, i y}=0 \quad \text { in } P, \\
& a_{i j}(\mathbf{y}) m_{, j y} n_{j}=0 \quad \text { at } S, \\
& m_{, j y} n_{j}=1 \quad \text { at } T, \\
& {\left[m_{, j y} n_{j}\right]=4 \text { at } S .}
\end{aligned}
$$

Numerical simulation of this problem has been performed in ANSYS, using the option "Heat gener(ation). Online". The quantity $|\nabla m(\mathbf{y})|$ is displayed on Figs. 5 and 6. One can see from Figs. 5 and 6 that the boundary layers are rapidly decaying. 


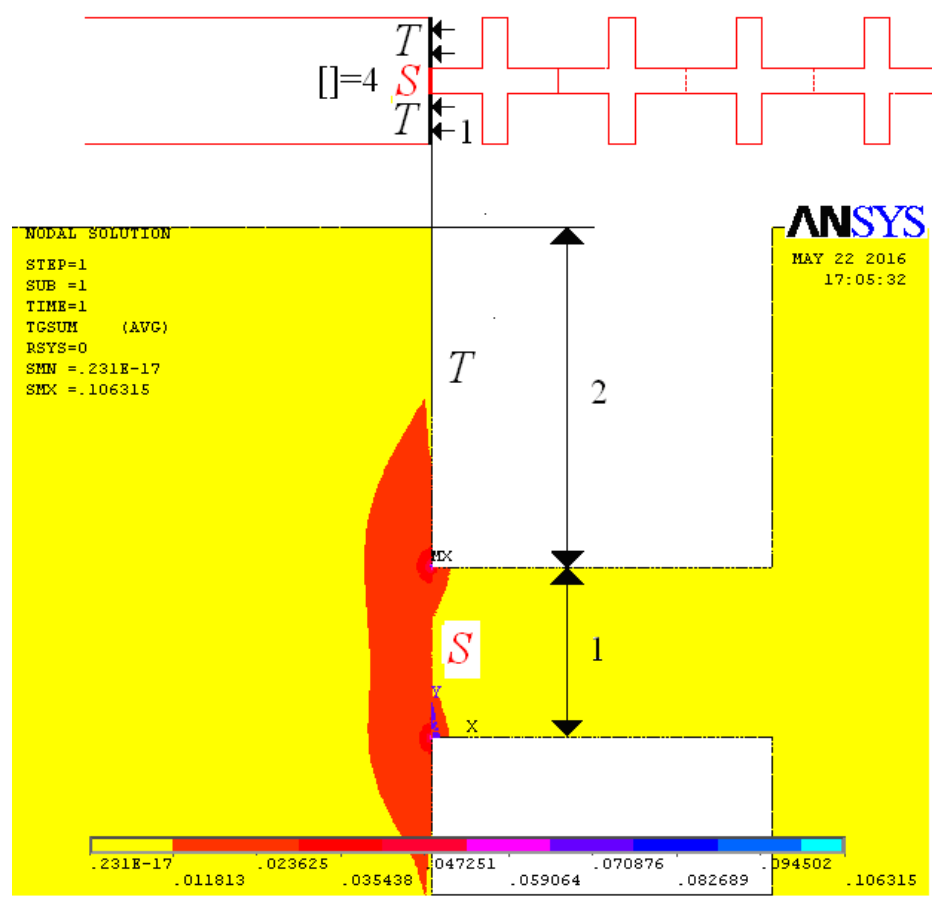

Figure 5. The quantity $|\nabla m(\mathbf{y})|$ on the joint.

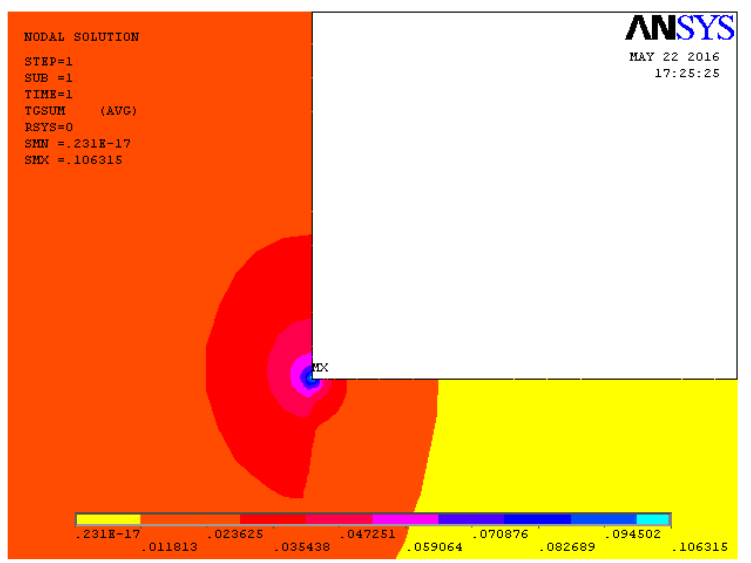

Figure 6. The quantity $|\nabla m(\mathbf{y})|$ (enlarged).

Let us now discuss the homogenization procedure for the flux, taking into account the jump in the derivative of the homogenized solution. First, we formulate the problem for the periodicity cell in terms of the flux. We stress again that the derivative $\frac{\partial u_{0}}{\partial x_{1}}$ is discontinuous. The problem (10) for the periodicity cell depends on the two functions, namely $u_{0,1 x}^{-}(\mathbf{x})$ and $u_{0,1 x}^{+}(\mathbf{x})$. Say, if the 
homogenized material is orthotropic, then $u_{0,1 x}^{ \pm}(\mathbf{x})=\frac{1}{A_{11}^{ \pm}} q_{1}^{(0) \pm}$, where $q_{1}^{(0) \pm}$ and $A_{i j}^{ \pm}$are the homogenized thermal fluxes and the homogenized coefficients of thermal conductivity in the left and right zones, respectively.

At the boundary between the left and right zones, the balance condition $q_{1}^{(0)+}=q_{1}^{(0)-}$ (here $q_{1}^{(0)-}$ and $q_{1}^{(0)+}$ are the flux values from the left and from the right of the interface, respectively) should be satisfied, meaning that the homogenized flux has no jump on the joint. Let us denote this value by $q_{1}^{(0)}$, then

$$
u_{0,1 x}^{ \pm}(\mathbf{x})=\frac{1}{A_{11}^{ \pm}} q_{1}^{(0)}(\mathbf{x})
$$

On substituting these expressions into (10), we obtain

$$
\begin{array}{ll}
\left(a_{i j}(\mathbf{y}) M_{, j y}+\frac{a_{i 1}(\mathbf{y})}{A_{11}^{-}} q_{1}^{(0)}(\mathbf{x}) \chi^{-}(\mathbf{x})+\frac{a_{i 1}(\mathbf{y})}{A_{11}^{+}} q_{1}^{(0)}(\mathbf{x}) \chi^{+}(\mathbf{x})\right)_{, i y}=0 & \text { in } P, \\
\left(a_{i j}(\mathbf{y}) M_{, j y}+\frac{a_{i 1}(\mathbf{y})}{A_{11}^{-}} q_{1}^{(0)}(\mathbf{x}) \chi^{-}(\mathbf{x})+\frac{a_{i 1}(\mathbf{y})}{A_{11}^{+}} q_{1}^{(0)}(\mathbf{x}) \chi^{+}(\mathbf{x})\right) n_{j}=0 & \text { at } S,
\end{array}
$$

where, once again, $M(\mathbf{y})$ is periodic with period 1 along $y_{2}$, tending to a periodic function with periods $m^{ \pm}$along $y_{1}$ in the limits $y_{1} \rightarrow \pm \infty$.

Since $q_{1}^{(0)}(\mathbf{x})$ has no jump, we can treat the homogenized thermal flux $q_{1}^{(0)}(\mathbf{x})$ in (18) as a parameter and carry out the usual multiple scale procedure of conventional homogenization theory. As a result, the solution of (18) can be presented in the form

$$
M=\mu(\mathbf{y}) q_{1}^{(0)}(\mathbf{x}),
$$

where

$$
\begin{aligned}
& \left(a_{i j}(\mathbf{y}) \mu_{, j y}+\frac{a_{i 1}(\mathbf{y})}{A_{11}^{-}} \chi^{-}(\mathbf{x})+\frac{a_{i 1}(\mathbf{y})}{A_{11}^{+}} \chi^{+}(\mathbf{x})\right)_{, i y}=0 \quad \text { in } P, \\
& \left(a_{i j}(\mathbf{y}) \mu_{, j y}+\frac{a_{i 1}(\mathbf{y})}{A_{11}^{-}} \chi^{-}(\mathbf{x})+\frac{a_{i 1}(\mathbf{y})}{A_{11}^{+}} \chi^{+}(\mathbf{x})\right) n_{j}=0 \quad \text { at } S,
\end{aligned}
$$

with $\mu(\mathbf{y})$ being periodic with period 1 along $y_{2}$ and tending to a periodic function with periods $m^{ \pm}$along $y_{1}$ in the limits $y_{1} \rightarrow \pm \infty$.

Let us now describe the procedure for correctors. The solution of (10) may be expressed as a sum of solutions corresponding to the terms $a_{i 1}(\mathbf{y}) \frac{\partial u_{0}(\mathbf{x})}{\partial x_{1}}$ and $a_{i 2}(\mathbf{y}) \frac{\partial u_{0}(\mathbf{x})}{\partial x_{2}}$. The solutions 
associated with these terms are $\mu(\mathbf{y}) q_{1}^{(0)}(\mathbf{x})$ and $N^{(2)}(\mathbf{y}) \frac{\partial u_{0}(\mathbf{x})}{\partial x_{2}}$, respectively. Here $N^{(2)}(\mathbf{y})$ is the solution of the problem for elementary cell, as introduced in the classical homogenization theory, see [1]. Thus, the corrector has the form

$$
\varepsilon u_{1}(\mathbf{x} / \varepsilon, \mathbf{x})=\varepsilon\left[\mu(\mathbf{x} / \varepsilon) q_{1}^{(0)}(\mathbf{x})+N^{(2)}(\mathbf{x} / \varepsilon) \frac{\partial u_{0}(\mathbf{x})}{\partial x_{2}}\right] .
$$

It should be noted that though the contribution of the corrector to temperature function is of order $\varepsilon$, the gradient of corrector $\varepsilon u_{1}(\mathbf{y})$ is of order unity. Thus, the leading order contribution of the corrector is

$$
\nabla_{x}\left[\varepsilon u_{1}(\mathbf{x} / \varepsilon, \mathbf{x})\right] \approx \nabla_{y} \mu(\mathbf{x} / \varepsilon) q_{1}^{(0)}(\mathbf{x})+\nabla_{y} N^{(2)}(\mathbf{x} / \varepsilon) \frac{\partial u_{0}(\mathbf{x})}{\partial x_{2}}
$$

We remark that the related problems of framed structures, including mathematical aspects of their homogenisation, have been considered, for example, in $[25,26]$.

\section{Correct joining of discrete (or originally continuous) and homogenized components}

The solution on the boundary may be written in terms of the homogenized values, whereas for the derivatives the effect of fast correctors should be taken into account. The solution $u_{\varepsilon}$ (temperature) for a real material is close to the homogenized solution $u_{0}(\mathbf{x})$. However, the derivatives and the associated temperature gradient and heat flux differ significantly from the derivatives and flux of the homogenized problem. The first and most evident distinction from the classical homogenization is that the derivatives $\nabla u_{\varepsilon}$ and the associated local fluxes in real material are considerably inhomogeneous on the micro-level, since they depend on the fast variable $\mathbf{y}$. At the same time, the gradients $\nabla u_{0}$ and fluxes, arising from the homogenized problem, are independent of the fast variable (except for the case described in section 2). The above stated may be written formally as

$$
\begin{gathered}
u_{\varepsilon} \approx u_{0}(\mathbf{x}), \\
\nabla u_{\varepsilon} \approx R\left(\mathbf{x}, \mathbf{y}, u_{0}(\mathbf{x})\right), \\
q_{\varepsilon} \approx T\left(\mathbf{x}, \mathbf{y}, u_{0}(\mathbf{x})\right)=a(\mathbf{y}) \nabla R\left(\mathbf{x}, \mathbf{y}, u_{0}(\mathbf{x})\right),
\end{gathered}
$$

where $T\left(\mathbf{x}, \mathbf{y}, u_{0}(\mathbf{x})\right)$ and $P\left(\mathbf{x}, \mathbf{y}, u_{0}(\mathbf{x})\right)$ are operators, providing a correspondence between the homogenized solution $u_{0}(\mathbf{x})$ or its gradient, and a function of the fast variable. 
The form of these operators depends on the particular class of the considered problems and is known for classical problems of mathematical physics. For example, for problem of heat transfer in periodic media we have

$$
R\left(\mathbf{x}, \mathbf{y}, u_{0}(\mathbf{x})\right)=\nabla_{y} N^{(k)}(\mathbf{y}) \frac{\partial u_{0}(\mathbf{x})}{\partial x_{k}},
$$

see [1].

As follows from the results of the conventional homogenization theory, correct approximation of solution of the original problem necessitates taking into consideration both homogenized components and fast correctors. In fact, the latter are crucial for correct joining of the bodies of different nature, as seen from a numerical experiment presented below.

Clearly, all physical bodies are inhomogeneous, if the consideration is performed on sufficiently small scale, with physical and mechanical properties varying during transition from level to level (e. g. metals considered on the levels of grains and molecules have different structure). From this point of view, all of the material characteristics (e.g. coefficient of heat transfer) are homogenized parameters. Thus, it follows that the material parameters of an inhomogeneous body depend on the level of investigation. Indeed, if the consideration is focused on macroscopic characteristics, then the classical material characteristics should be used. On the other hand, investigation of micro-structural behavior, e.g. joining the micro- and macrostructure fragments requires not only knowledge of the classical material parameters, but also of the operators $R\left(\mathbf{x}, \mathbf{y}, u_{0}(\mathbf{x})\right)$ and $T\left(\mathbf{x}, \mathbf{y}, u_{0}(\mathbf{x})\right)$, which are the material characteristics of the macromaterial governed by its microstructure.

For many classical linear problems the operators $R\left(\mathbf{x}, \mathbf{y}, u_{0}(\mathbf{x})\right)$ and $T\left(\mathbf{x}, \mathbf{y}, u_{0}(\mathbf{x})\right)$ take the form

$$
\begin{aligned}
& R\left(\mathbf{x}, \mathbf{y}, u_{0}(\mathbf{x})\right)=r(\mathbf{y}): \nabla u_{0}(\mathbf{x}), \\
& T\left(\mathbf{x}, \mathbf{y}, u_{0}(\mathbf{x})\right)=p(\mathbf{y}): \nabla u_{0}(\mathbf{x}),
\end{aligned}
$$

with colon denoting tensor convolution.

The operators $r(\mathbf{y})$ and $p(\mathbf{y})$ are sometimes referred to as concentration tensors, since they describe the microscopic flux/gradient field within a macroscopic material fragment subjected to the homogeneous macroscopic field of unit gradient. Therefore, the concentration tensors of the microscopic flux/gradient should be taken into consideration as material characteristics for analysis on the micro-level.

Let us clarify the discussion above. Indeed, various models of microstructure are adopted by researchers when joining molecular structures with homogeneous bodies. All of these models are inhomogeneous, including even the recent non-local models [27]. When joining the material 
described by such a model with the homogeneous media, not only the knowledge of homogenized characteristics is required, but also that of micro-inhomogeneous, or even micro-non-local response of the homogeneous material caused by macroscopic loading.

Consider now the laminated material containing isotropic layers of the same width with dimensionless heat transfer coefficients of 1 and 10. Let us imagine the material separated into two parts, and the left half replaced by the homogenized (orthotropic) material, with the dimensionless heat transfer coefficientsin the $O x$ - and $O y$-axis directions being equal to 5.5 and 1.82, respectively. The elementary cell is shown on Fig. 7 (bottom). Now let us join the original (laminated) and the homogenized parts.

The conditions on the interface between the layered and homogeneous materials are

$$
\begin{gathered}
{[m(\mathbf{y})]=0,} \\
{\left[a_{i j}(\mathbf{y}) m_{, j y}\right] n_{j}=\left(a_{i j}(\mathbf{y}) N_{, j y}^{k+}(\mathbf{y}) u_{0, k x}^{+}(\mathbf{x})-a_{i j}(\mathbf{y}) N_{, j y}^{k-}(\mathbf{y}) u_{0, k x}^{-}(\mathbf{x})\right) n_{j} .}
\end{gathered}
$$

In case of a homogeneous material, the derivatives from the left of the solution for the elementary cell are given by $N_{, j y}^{k-}(\mathbf{y})=\delta_{j k}$, where $\delta_{j k}$ is the Kronecker delta. Since the averaged constants of the right and left parts are the same, and from the global point of view both halves form a homogeneous body, the solution $u_{0}(\mathbf{x})$ of the averaged problem should be continuous together with the derivatives. Then, on the interface we have $u_{0,1 x}^{+}=u_{0,1 x}^{-}$. As a result, equation (25) may be rewritten in the form

$$
\left[a_{i j}(\mathbf{y}) m_{, j y}\right] n_{j}=\left(a_{i j}(\mathbf{y}) N_{, j y}^{k+}(\mathbf{y})-A_{i k}\right) u_{0, k x}(\mathbf{x}) n_{j} .
$$

Note that the right hand side of (26) is not zero, which means emergence of a boundary layer.

On the other hand, if the layered material is joined with the corresponding homogenized material (i.e. fictitiously homogeneous), then the actual flux in the homogenized material will be $a_{i j}(\mathbf{y}) N_{, j y}^{1-}(\mathbf{y}) u_{0,1 x}^{-}(\mathbf{x})$, rather than $A_{i j} u_{0,1 x}(\mathbf{x})$. So, instead of (26), condition (25) should be used. In this case the microstructure of the left and right parts are identical, so are the solutions for elementary cells: $N^{-}(\mathbf{y})=N^{+}(\mathbf{y})$. Hence, the right hand side of (25) is zero and the boundary layer does not appear.

A natural question occurs, namely, since all real materials are inhomogeneous, does it mean, that the boundary layer emerges in every contact of different materials? This study gives a positive answer. However, we note that the width of this boundary layer is of order of typical size of inhomogeneity, so during the contact of two homogeneous crystal bodies the width of the boundary layer is of order of a typical crystal size. 
The results of computations of the boundary layer, arising in the problem for an elementary cell (14) are displayed in Fig. 7 (top). It is clear that the boundary layer emerges, if the material on the left is a real homogeneous material. It is also obvious, that if a part of laminated material is first homogenized and then joined with the remaining part of the laminated material, then there should be no boundary layers arising, i.e. if a boundary layer is observed, it can only be a defect of the methodology. The consistent matching of the homogenized and the corresponding laminated materials should then be performed according to the methodology described above. The procedure explained above in Sect. 3 leads to coupling with no boundary layer.

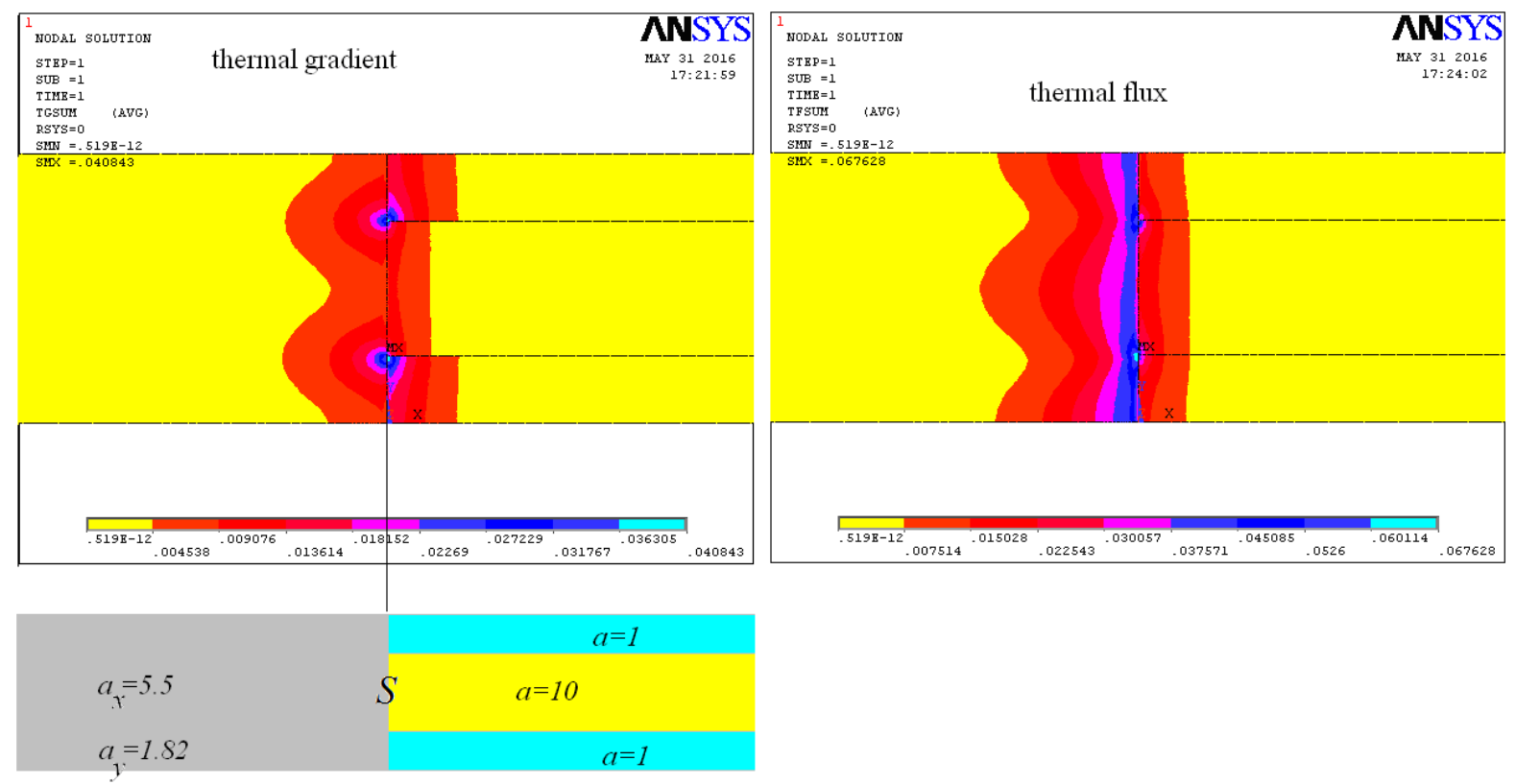

Figure 7. Boundary layer on the interface of a layered material and the homogenized one.

We finalize the consideration in this paper with an example of a boundary layer arising in the vicinity of the interface of two structures, which possess the same homogenized characteristics but have different microstructures. Consider a body, assembled of fragments with homothetic similar microstructure $\varepsilon P_{0}=\left\{\varepsilon \mathbf{x}: \mathbf{x} \in P_{0}\right\}$, which may be constructed as follows. First, we consider a periodic structure with periodicity cell $P_{0}$. After that, we construct a body with the right part $x_{1}>0$ having periodic structure with the periodicity cell $P^{+}=\varepsilon_{2} P_{0}$ and left part $x_{1}<0$ having periodic structure with the periodicity cell $P^{-}=\varepsilon_{1} P_{0}$, see Fig. 8 (top). For the case displayed in Fig. $8, P_{0}$ is the structure with periodically distributed circular holes of unit radius. 


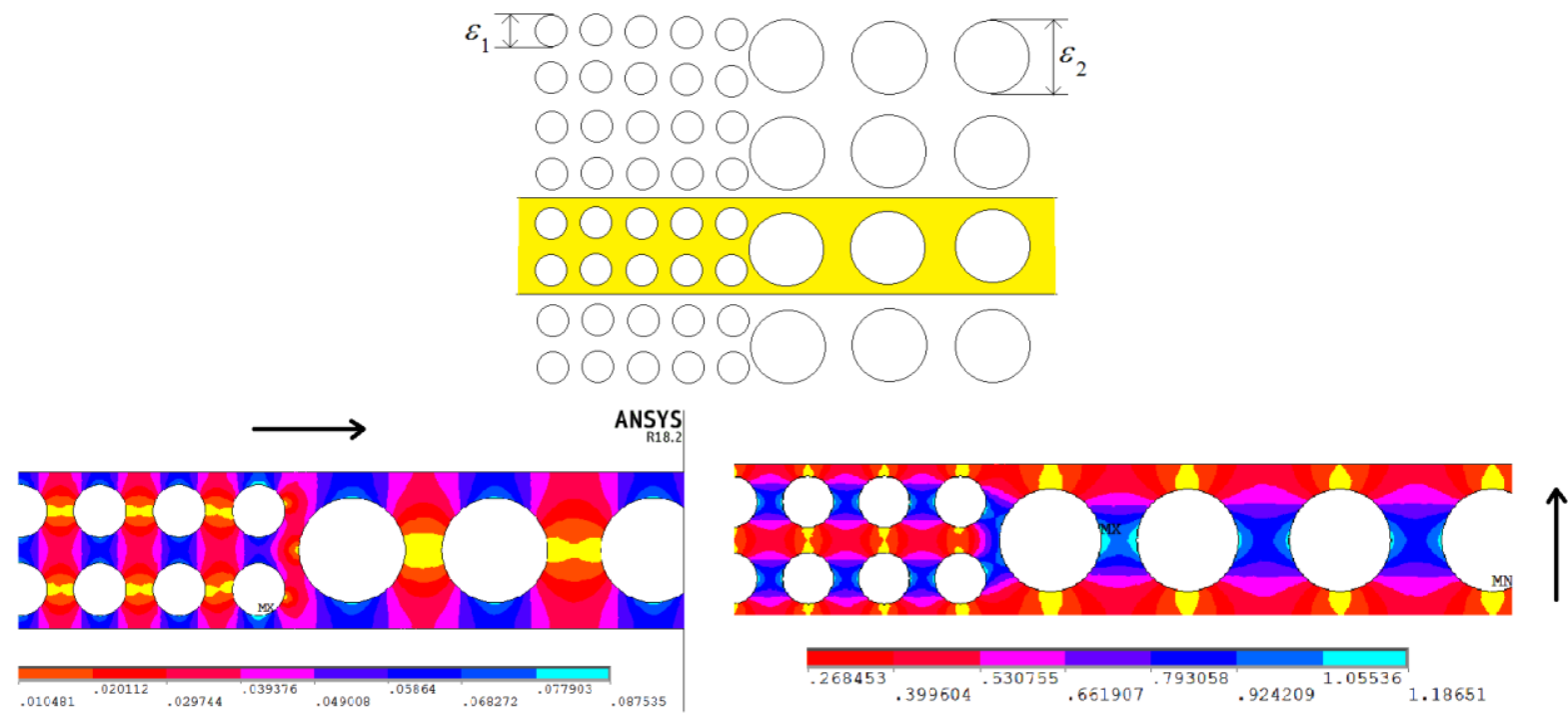

Figure 8. Joining of two bodies with homothetic similar microstructures and its periodicity cell (top). Gradient of solution $N^{1}(\mathbf{y})$ and $N^{2}(\mathbf{y})$ of the periodicity cell problem (bottom).

The bodies with homothetic similar microstructures have equal homogenized characteristics. In other words, from macroscopic point of view, the left and right parts form a homogeneous body. Solution $u_{0}(\mathbf{x})$ of the macroscopic problem of heat transfer for this body is smooth (without jumps in the derivatives). At the same time, if $\varepsilon_{1} \neq \varepsilon_{2}$, the microstructures of the left and right components are different, though solutions of the periodicity cell problems are the same in view of the homothetic similarity of the microstructure.

Let us denote this solution by $N^{k}(\mathbf{y})$. In this notation, the right-hand side of (25) becomes $a_{i j}(\mathbf{y})\left(N_{, j y}^{k}\left(\mathbf{x} / \varepsilon_{1}\right)-N_{, j y}^{k}\left(\mathbf{x} / \varepsilon_{2}\right)\right) u_{0, k x}(\mathbf{x}) n_{j}$. This function is not equal to zero, implying boundary layers appearing in the vicinity of $x_{1}=0$, compensating for this jump of correctors.

Fig. 8 (bottom) displays gradients of solution of the periodicity cell problem, $N^{1}(\mathbf{y})$ and $N^{2}(\mathbf{y})$, with the arrows indicating the direction of the overall (macroscopic) field. The perturbation of the periodic field in the contact zone is clearly seen. It should be emphasized once again, that the material displayed in Fig. 8 is homogeneous when considered from the macroscopic point of view.

\section{Conclusion}

Application of homogenization theory to macro-inhomogeneous bodies or structures containing fragments which cannot be homogenized has some peculiarities. A natural theory describing this 
process is interesting from both theoretical and practical points of view, with the consideration in this paper carried out for the heat equation. The major focus of this study is on the boundary layer arising over the transition region localized in the vicinity of the joint. The challenge of the studied problem is, whether this boundary layer emerges naturally due to underlying physics, or it is a spurious one, appearing due to flaws in the methodology.

In particular, it is demonstrated that the procedure of joining of two homogenized bodies should take into consideration the rapidly varying correctors, which guarantees absence of fictitious boundary layers. It is suggested that for micro-structural problems the set of classical material characteristics should be complemented by the operators describing the microscopic response of the macroscopic body to macroscopic deformations.

The formulation of the problem for the periodicity cell is presented for the case when the homogenized flux fields possess discontinuities (when these cannot be treated as independent of the fast variables). The construction of the first order correctors is also described. The theoretical formulation is confirmed by numerical simulations.

The problems considered in detail include boundary layers emerging on the interface (contact zone) in case of bodies with different averaged characteristics, as well as bodies with the same averaged characteristics, but different microstructure. In addition, a physically correct formulation of the condition on the interface between the fragment of the original material and the corresponding homogenized material, which does not lead to fictitious boundary layers, is presented.

The developed formal scheme, aiming at a physical insight into the nature of boundary layers, does not involve a more rigorous mathematical justification, including, for example, evaluation of the asymptotic remainder, which should be a subject of special consideration. At the same time, it may be shown that the exact solution for a particular case of the uniform flux fields within the homogenized components confirms all of the findings in this paper.

The obtained results extend further the ideas presented in [28, 29], and may find their applications in engineering practice $[30,31]$. Using these results, it is possible to determine whether the boundary layer arises on the interface of materials of different structure. Estimates for width and intensity of the boundary layer could also be obtained.

\section{Acknowledgement}

The work of AGK was supported by the London Mathematical Society (Ref. 21513), and also by Keele University, UK. Fruitful discussions with J. Kaplunov are also gratefully acknowledged. The authors thank S. I. Rakin for assistance in numerical computations. 


\section{References}

1. E. Sanchez-Palencia, Non-Homogeneous Media and Vibration Theory (Lect. Notes in Physics 127, Springer-Verlag, Berlin, 1980).

2. H. Ben Dhia and G. Rateau, The Arlequin method as a flexible engineering design tool. Int. J. Numer. Met. Eng. 62 (2005)1442-1462.

3. H. Ben Dhia, Further insights by theoretical investigations of the multiscale Arlequin method. Int. J. Multisc. Comp. Eng. 6 (2008) 215-232.

4. G. Lubineau, Y. Azdoud, F. Han, C. Rey and A. Askari, A morphing strategy to couple nonlocal to local continuum mechanics. J. Mech. Phys. Solids 60 (2012) 1088-1102.

5. T. Bauman, H. Ben Dhia, N. Elkhodja, J. T. Oden and S. Prudhomme, On the application of the Arlequin method to the coupling of particle and continuum models. Comp. Mech. 42 (2008) 511-530.

6. S. Pfaller, G. Possart, P. Steinmann, M. Rahimi, F. Müller-Plathe and M. C. Böhm, A comparison of staggered solution schemes for coupled particle-continuum systems modeled with the Arlequin method. Comp. Mech. 49 (2012) 565-579.

7. G. J. Wagner and W. K. Liu, Coupling of atomistic and continuum simulations using a bridging scale decomposition. J. Comp. Phys. 190 (2003) 249-274.

8. S. P. Xiao and T. Belytschko, A bridging domain method for coupling continua with molecular dynamics. Comp. Met. Appl. Mech. Eng. 193 (2004) 1645-1669.

9. M. L. Parks, P. B. Bochev and R. B. Lehoucq, Connecting atomistic-to-continuum coupling and domain decomposition. Multisc. Mod. Simul. 7 (2008) 362-380.

10. H. Ben Dhia, Global-local approaches: the Arlequin framework. Europ. J. Comp. Mech. 15 (2006) 67-80.

11. H. Hu, N. Damil and M. Potier-Ferry, A bridging technique to analyze the influence of boundary conditions on instability patterns. J. Comp. Phys. 230 (2011) 3753-3764.

12. B. Smith, P. Bjørstad and W. Gropp, Domain Decomposition (Cambridge University Press, 1996).

13. I. V. Andrianov, V. V. Danishevs'kyy and D. Weichert, Analytical study of the load transfer in fibre-reinforced 2D composite materials. Int. J. Solids Struct. 45 (2008) 1217-1243.

14. V. Ehrlacher, C. Ortner and A. V. Shapeev, Analysis of boundary conditions for crystal defect atomistic simulations. arXiv:1306. 5334v3 [math. NA], 14 July 2015.

15. I. V. Andrianov, V. V. Danishevs'kyy and A. L. Kalamkarov, Vibration localization in onedimensional linear and nonlinear lattices: discrete and continuum models. Nonlin. Dyn. $\mathbf{7 2}$ (2013) 37-48.

16. I. V. Andrianov, V. V. Danishevs'kyy and I. A. Kushnierov, Spatial localization of linear elastic waves in composite materials with defects. Z. Angew. Mat. Mech. 94 (2014) 10011010 .

17. A. Matine, N. Boyard, P. Cartraud, G. Legrain and Y. Jarny, Modeling of thermophysical properties in heterogeneous periodic media according to a multi-scale approach: Effective conductivity tensor and edge effects. Int. J. Heat Mass Transfer 62 (2013) 586-603.

18. G. P. Panasenko, Higher order asymptotics of solutions of problems on the contact of periodic structures. Math. USSR-Sbornik 38 (1981) 465-494.

19. N. S. Bakhvalovand G. P. Panasenko, Averaging Processes in Periodic media. Mathematical problems in mechanics of composite materials. (Kluwer, Dordrecht, 1989).

20. G. P. Panasenko, Multi-Scale Modeling for Structures and Composites (Springer, Berlin, 2005).

21. V. A. Kozlov, V. G. Maz'ya and A. B. Movchan, Asymptotic Analysis of Fields in MultiStructures (Clarendon Press, Oxford, 1999).

22. V. A. Kozlov, V. G. Maz'ya and A. B. Movchan, Fields in non-degenerate 1D-3D elastic multistructures. Quart. J. Mech. Appl. Math. 54 (2001) 177-212. 
23. O. A. Ladyzhenskaya, V. Y. Rivkind and N. N. Ural'tseva, The classical solvability of diffraction problems. In: Boundary value problems of mathematical physics. Trudy Steklov Mat. Inst. 92 (1966) 116-146.

24. O. A. Ladyzhenskaya and N. N. Ural'tseva, Linear and Quasilinear Elliptic Equations (Mathematics in Science and Engineering, Vol. 46, Academic Press, NY, London, 1968).

25. A. Hrennikoff, Solution of problems in elasticity by the framework method. J . Appl. Mech., 6, 1941, 169-175.

26. P. G. Martinsson and I. Babuška, Mechanics of materials with periodic truss or frame microstructures. Arch. Rational Mech. Anal. 185 (2007) 201-234.

27. A. V. Shapeev, Consistent energy-based atomistic/continuum coupling for two-body potentials in one and two dimensions. arXiv:1010.0512v3 [math. NA] 8 Aug 2011.

28. A. G. Kolpakov and A. Gaudiello, Influence of non degenerated joint on the global and local behavior of joined rods. Int. J. Eng. Sci. 49 (2011) 295-309.

29. A. L. Kolpakov and I. V. Andrianov, Asymptotic decomposition in the problem of joined elastic beams. Z. Angew. Mat. Mech. 94 (2014) 818-836.

30. A. G. Kolpakov, S. I. Rakin, Estimation of Stress Concentration in a Welded Joint Formed by Explosive Welding. J. Appl. Mech. Tech. Phys., 59(3) (2018) 569-575.

31. A. G. Kolpakov, I. V. Andrianov, S. I. Rakin, G. A. Rogerson, An asymptotic strategy to couple homogenized elastic structures. Int. J. Eng. Sci. 131 (2018) 26-39. 